\title{
A gyrocompass for maritime applications based upon multivariable control theory
}

\author{
OLAV EGELAND
}

\begin{abstract}
Keywords: gyrocompass, maritime navigation, Kalman filtering, parameter estimation.

A gyrocompass is designed using multivariable control theory. The compass can be implemented with an inertial platform or as a strap-down system. Measurement noise caused by vessel acceleration is modeled and feedforward is taken from vessel speed. Though the model is of order 9, it has only three unknown parameters of which one can be chosen a priori. Parameter estimation is discussed. For simulation of the compass, a non-linear surface vessel model with 6 degrees of freedom and wave excitation is used.
\end{abstract}

\section{Introduction}

In maritime navigation the need arises for an accurate compass that is not disturbed by magnetic fields. A compass that satisfies these requirements is the gyrocompass, which indicates north by measuring angular velocity and the direction of the specific force. The specific force is acceleration minus gravity.

A maritime gyrocompass should be designed to indicate north in the presence of vessel accelerations, and it should not be dependent on velocity or position measurements.

Traditionally, maritime gyrocompasses have been made with a gyroscope with two degrees of freedom, mechanical feedback and hydraulic damping (Wrigley et al. 1969). The Sperry and Anschutz compasses are of this type. These compasses are sensitive to vessel acceleration. Alignment is slow, as the compass error can be described as a damped oscillation with a period of $84 \mathrm{~min}$.

A better compass can be made by using an inertial platform (Wrigley et al. 1969) with two gyros with one degree of freedom and two pendulums. By means of a digital control system, feedforward from vessel speed and even the centrifugal forces can be implemented, and multivariable control theory can be applied in the place of the conventional Schuler-tuning in two decoupled loops (Wrigley et al. 1969).

Instead of an inertial platform system, a strap-down system can be used (Schmidt 1978). Here, the sensors are mounted directly on the vessel. To instrument a gyrocompass as a strap-down system, three gyros with one degree of freedom and three pendulums are required.

Compared to the conventional gyrocompass, these more advanced compasses are more expensive, but due to the recent development in sensors and low-cost computing power they are interesting.

The fibre-optic laser gyro (Ezekiel and Knaussenberge 1978) has the potential to become significantly cheaper than traditional mechanical gyros of the same accu-

Received 18 December 1984; revised 1 February 1985.

$\dagger$ Division of Engineering Cybernetics, University of Trondheim, The Norwegian Institute of Technology, N-7034 Trondheim-NTH, Norway. 
racy. This will make strap-down systems especially interesting as such a system would have no moving parts, which may increase the reliability of the system.

An inertial navigation system (Britting 1971, Leondes 1970, Maybeck 1979) is a very accurate north indicator. Such a system requires accelerometers instead of pendulums and three gyros with one degree of freedom also in the platform case, and measurements of vessel velocity or position are needed to dampen the system.

In this paper, a multivariable,stochastic control system is designed for a gyrocompass implemented as a platform system or as a strap-down system. In $\S 2$ stochastic models of the compass and the environment are presented. Estimation and control are discussed in $\S 3$, and parameter estimation is discussed in $\S 4$. The compass is simulated with a vessel model with six degrees of freedom and wave excitation in $\S 5$.

\section{Modeling}

\subsection{The instrumented coordinate frame}

In a gyrocompass, we want to align the instrumented coordinate frame with the local level frame which has axes in the north, east and down direction. The instrumented axis aligned with the north axis will then indicate north.

A coordinate frame can be instrumented with an inertial platform. In an inertial platform, the sensors are mounted on a controlled member which is torqued with a commanded inertial angular velocity $\omega_{\text {ref }}$ (Fig. 1). The controlled member is suspended in gimbals controlled by torque-engines. Feedback is taken from the gyroscopes, which are inertial angular velocity sensors.

In a strap-down system, a coordinate frame is instrumented by computing the coordinate transformation matrix from the vessel frame to the instrumented frame. This is done using gyro measurements.

We now consider a gyrocompass with an inertial platform with three gyros with one degree of freedom or a strap-down instrumentation. The only difference between the mathematical models for these two systems is the effect of component noise, which is discussed in $\S 2.4$.

We denote the inertial coordinate frame by $i$, the earth frame by $e$, the local level frame by $n$, and the instrumented frame by $k$.

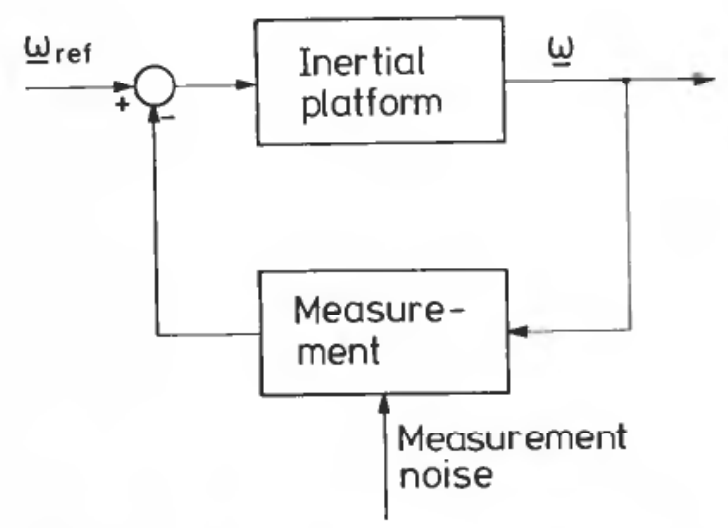

Figure 1. Block diagram of inertial platform. 
The $k$ frame is nominally aligned with the $n$ frame. The $k$ frame has an inertial angular velocity

$$
\omega_{i k}=u+v_{p}
$$

where $\omega_{i k}$ is the angular velocity of the $k$ frame relative to the $i$ frame, $u$ is the control vector applied to the inertial platform and $v_{p}$ is process noise in the inertial platform. The dominant term in $\boldsymbol{v}_{p}$ is the gyro measurement noise.

Generally, the transformation between two coordinate frames is given by three Euler angles. For infinitesimal rotations, the order of the rotations need not be specified. We therefore define a vector $\boldsymbol{\theta}$ of the angles between the $k$ and $n$ frame assuming that the angles are small. We now have

$$
\frac{d}{d t_{i}} \boldsymbol{\theta}=\omega_{n k}
$$

where subscript $i$ on the time differentiation operator denotes differentiation in the $i$ frame. $\omega_{n k}$ is given by

Now

$$
\omega_{n k}=\omega_{i k}-\omega_{i n}
$$

$$
\frac{d}{d t_{i}} \mathbf{0}-\frac{d}{d t_{n}} \boldsymbol{\theta}+\boldsymbol{\omega}_{\text {in }} \times \boldsymbol{\theta}
$$

where $x$ is the vector cross-product operator. Combining (2)-(5) we get

$$
\frac{d}{d t_{n}} \boldsymbol{\theta}=-\boldsymbol{\omega}_{\text {in }} \times \boldsymbol{\theta}+\boldsymbol{u}-\boldsymbol{\omega}_{\text {in }}+\boldsymbol{v}_{p}
$$

We now coordinatize (6) in the $n$ frame. We let $x_{1}=\left[\theta_{\mathrm{N}}, \theta_{\mathrm{E}}, \theta_{\mathrm{D}}\right]^{T}$ where $\theta_{\mathrm{N}}, \theta_{\mathrm{E}}$ and $\theta_{\mathrm{D}}$ are the components of $\theta$ in north, east and down direction (Fig. 2). Further,

$$
\omega_{i n}^{n}=\left[\begin{array}{c}
\left(\omega_{i e}+l\right) \cos L \\
-\dot{L} \\
-\left(\omega_{i e}+l\right) \sin L
\end{array}\right]=\left[\begin{array}{l}
\Omega_{\mathrm{N}} \\
\Omega_{\mathrm{E}} \\
\Omega_{\mathrm{D}}
\end{array}\right]
$$

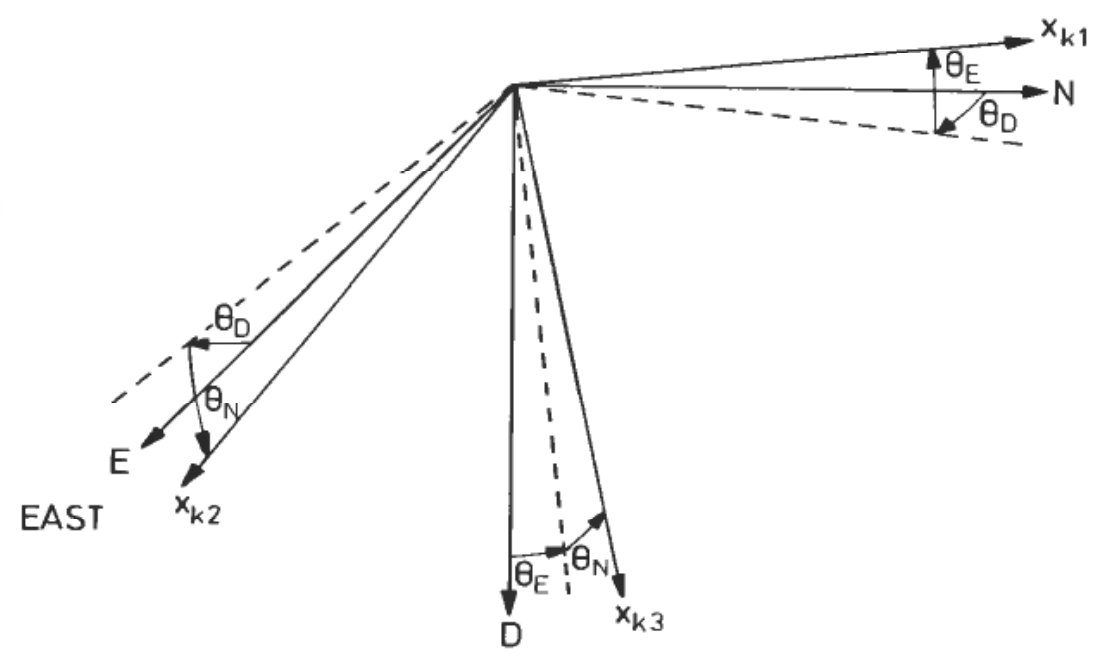

DOWN

Figure 2. Definition of angles $\theta_{\mathrm{N}}, \theta_{\mathrm{E}}$ and $\theta_{\mathrm{D}}$. 
where $\omega_{i n}^{n}$ is $\omega_{i n}$ coordinatized in the $n$ frame, $\omega_{i e}$ is the magnitude of the earth's angular velocity, $l$ is longitude and $L$ is latitude. This gives

$$
\dot{x}_{1}=A_{11} x_{1}+u+v
$$

where

$$
A_{11}=\left[\begin{array}{rcr}
0 & \Omega_{\mathrm{D}} & -\Omega_{\mathrm{E}} \\
-\Omega_{\mathrm{D}} & 0 & \Omega_{\mathrm{N}} \\
\Omega_{\mathrm{E}} & -\Omega_{\mathrm{N}} & 0
\end{array}\right]
$$

and

$$
v=\left[\begin{array}{l}
-\Omega_{\mathrm{N}} \\
-\Omega_{\mathrm{E}} \\
-\Omega_{\mathrm{D}}
\end{array}\right]+v_{p}
$$

\subsection{Measurement model}

The direction of the specific force $f$ relative to the $k$ frame is measured by pendulums. $f$ is given by

$$
\boldsymbol{f}=\boldsymbol{a}-\boldsymbol{g}
$$

where $\boldsymbol{a}$ is acceleration and $\boldsymbol{g}$ is gravity. Coordinatized in the $n$ frame, $f$ can be approximated by

$$
\boldsymbol{f}^{n}=\left[\begin{array}{l}
a_{\mathrm{N}} \\
a_{\mathrm{E}} \\
a_{\mathrm{D}}
\end{array}\right]+\left[\begin{array}{r}
0 \\
0 \\
-g
\end{array}\right]
$$

where $a_{\mathrm{N}}, a_{\mathrm{E}}$ and $a_{\mathrm{D}}$ are the components of $a$ in the north, east and down direction. Assuming the angles $x_{1}, x_{2}$ and $x_{3}$ to be small, we have

$$
f^{k}=\left[\begin{array}{ccc}
1 & x_{3} & -x_{2} \\
-x_{3} & 1 & x_{1} \\
x_{2} & -x_{1} & 1
\end{array}\right] \boldsymbol{f}^{n}
$$

where $\boldsymbol{f}^{k}$ is coordinatized in the $k$ frame. The measurements are approximated by

$$
\boldsymbol{y}=\left[\begin{array}{lll}
g & 0 & 0 \\
0 & g & 0
\end{array}\right] \boldsymbol{x}_{1}+\left[\begin{array}{r}
-a_{\mathrm{E}} \\
a_{\mathrm{N}}
\end{array}\right]+\boldsymbol{w}^{\prime}
$$

where $\boldsymbol{w}^{\prime}$ is measurement noise.

\subsection{Process noise}

As stated above, the dominant term in $v_{p}$ in (8) is the gyro measurement noise or, as it is more commonly called, the gyro drift.

We see from (6) that a stationary gyro drift, $v_{20}$, in the east direction causes a stationary compass error given by

$$
\theta_{\mathrm{Do}}=-v_{20} / \Omega_{\mathrm{N}} \text {. }
$$

This means a compass error of 2 mrad per meru drift where 1 meru is a milli earth rate unit. 
For a laser gyro, the drift can be modeled as a slowly changing bias plus white noise (Maybeck 1979). A slowly changing bias can be modeled as a Wiener process. Unfortunately, a state presenting the bias in the east direction would be unobservable.

For a platform system, we therefore model the gyro drift as

$$
\begin{array}{r}
\boldsymbol{v}_{p}=\left[\begin{array}{c}
x_{4} \\
0 \\
x_{5}
\end{array}\right]+\left[\begin{array}{l}
n_{1} \\
n_{2} \\
n_{3}
\end{array}\right] \\
{\left[\begin{array}{c}
\dot{x}_{4} \\
\dot{x}_{5}
\end{array}\right]=\left[\begin{array}{l}
n_{4} \\
n_{5}
\end{array}\right]}
\end{array}
$$

where $n_{1}, \ldots, n_{5}$ are independent noise processes. To get an estimate of east gyro drift, the platform can be rotated $90^{\circ}$ about the down axis when the north gyro drift estimate has converged (Brock and Schmidt 1970). We will then have a calibrated gyro in the east direction.

For a strap-down system, the model will be slightly more complicated because the gyros are mounted directly on the vessel. Here, both nominally horizontal gyros will contribute to the north drift. After some changes of course, an estimate of both gyro drifts can be made.

\subsection{Measurement noise modeling}

In the measurement model, acceleration in the north and east direction enters as noise. The classical approach to this problem is Schuler-tuning in mono-variable loops (Wrigley et al. 1969).

An accurate model of vessel accelerations would be non-linear, of high order and with many unknown parameters. We therefore seek an approximate model which satisfies our requirements.

The vessel accelerations consist of wave-induced accelerations and accelerations due to speed or course corrections.

The vessel acceleration due to course corrections can be compensated by feedforward from the gyros and the log. Wavc-induced accelerations and accelerations due to speed corrections must be modeled.

An acceleration model that is often used is

$$
\dot{a}=-\frac{1}{T} a+n
$$

where $n$ is white noise. In this problem, this acceleration model is not adequate. We notice that the stationary value of the acceleration is non-zero. For a surface vessel this is highly unrealistic. Also, this model will give an acceleration component in the range of frequencies of the state vector $x_{1}$, i.e. around the Schuler frequency ( $84 \mathrm{~min}$ oscillations).

We should therefore choose a second-order model

$$
\begin{aligned}
& \frac{a_{\mathrm{NW}}}{n_{\mathrm{N}}}(s)=\frac{s}{s^{2}+2 \zeta \omega_{0} s+\omega_{0}^{2}} \\
& \frac{a_{\mathrm{EW}}}{n_{\mathrm{E}}}(s)=\frac{s}{s^{2}+2 \zeta \omega_{0} s+\omega_{0}^{2}}
\end{aligned}
$$


where $a_{\mathrm{NW}}(s)$ and $a_{\mathrm{EW}}(s)$ are wave induced accelerations in the north and east direction, respectively, and $n_{\mathrm{E}}$ and $n_{\mathrm{N}}$ are independent white noise processes. Here, we have assumed north and east acceleration to be uncorrelated, as this gives a timeinvariant system. A more accurate model is hard to find for a general vessel. The natural frequency $\omega_{0}$ and the damping ratio $\zeta$ are dependent on vessel parameters. This model has a zero at the origin, and the stationary component of $a_{\mathrm{NW}}$ and $a_{\mathrm{EW}}$ is therefore zero.

\subsection{Vessel}

For simulation of the compass, a 6-degree-of-freedom non-linear model of a surface vessel is developed. The vessel is excited by an ITTC wave-spectrum (Price et al. 1974). The wave forces and moments are calculated for a block-shaped hull as in Blanke (1981). The vessel is equipped with a PD autopilot.

\section{Estimation and control}

The system is discretized by the Euler method with some modifications in $\Phi_{B}$ and $\Omega_{B}$, and sampling time $T$ is chosen as 1 second. The resulting system is

$$
\begin{aligned}
& \boldsymbol{x}(k+1)=\boldsymbol{\Phi} \boldsymbol{x}(k)+\Delta u(k)+\overline{\boldsymbol{v}}(k)+\boldsymbol{\Omega} \boldsymbol{n}(k) \\
& \boldsymbol{y}(k)=D x(k)+\boldsymbol{w}(k) \\
& \boldsymbol{x}^{T}=\left[\theta_{\mathrm{N}}, \theta_{\mathrm{E}}, \theta_{\mathrm{D}}, x_{4}, x_{5}, a_{\mathrm{NB}}, x_{7}, a_{\mathrm{NE}}, x_{9}\right] \\
& \Phi=\left[\begin{array}{c:cc:c:c} 
& T & 0 & & \\
\Phi_{11} & 0 & 0 & 0 & 0 \\
& 0 & T & & \\
\hdashline 0 & I_{2} & 0 & 0 \\
\hdashline 0 & 0 & \Phi_{B} & 0 \\
\hdashline 0 & 0 & 0 & \Phi_{B}
\end{array}\right] \\
& \Phi_{11}=I+A_{11} T \\
& \Phi_{B}=\left[\begin{array}{cc}
1 & T \\
-\omega_{0}^{2} T & 1-2 \zeta \omega_{0}-\omega_{0}^{2} T^{2}
\end{array}\right] \\
& \boldsymbol{u}=\left[u_{1}, u_{2}, u_{3}\right]^{T} \\
& \Delta=\left[\begin{array}{c:c}
I_{3} & 0 \\
\hdashline 0 & 0
\end{array}\right] \\
& \bar{v}(k)=\left[\begin{array}{c}
T \omega_{i n}^{n} \\
-0
\end{array}\right]=\left[\begin{array}{c}
v_{0} \\
\overline{0}
\end{array}\right] \\
& \Omega=\left[\begin{array}{c:c:c}
I_{5} & 0 & 0 \\
\hdashline 0 & \Omega_{B} & 0 \\
\hdashline 0 & 0 & \Omega_{B}
\end{array}\right]
\end{aligned}
$$




$$
\begin{gathered}
\boldsymbol{\Omega}_{B}=\left[\begin{array}{c}
1 \\
-\left(2 \zeta \omega_{0}+\omega_{0}^{2} T\right)
\end{array}\right] \\
\mathrm{D}=\left[\begin{array}{lllllllrl}
g & 0 & 0 & 0 & 0 & 0 & 0 & -1 & 0 \\
0 & g & 0 & 0 & 0 & 1 & 0 & 0 & 0
\end{array}\right]
\end{gathered}
$$

Process noise is $\boldsymbol{n}$ with covariance

$$
\begin{gathered}
\mathrm{E}\left[n(k) n(l)^{T}\right]=N \delta_{k l} \\
\mathrm{~N}=\operatorname{diag}\left(n_{11}, \ldots, n_{55}\right)
\end{gathered}
$$

and measurement noise is $w$ with covariance

$$
\begin{aligned}
& E\left[w(k) w(l)^{T}\right]=W \delta_{k l} \\
& \mathrm{~W}=\operatorname{diag}\left(w_{11}, w_{22}\right)
\end{aligned}
$$

The model is linear and time-invariant and there are only three unknown parameters, namely, latitude $L$ and two wave-motion parameters $\zeta$ and $\omega_{0}^{2}$, even though the model is of order 9 .

The Kalman filter equations are (Maybeck 1979)

$$
\begin{aligned}
\bar{x}(k) & =\bar{x}(k)+K(k)[y(k)-D \bar{x}(k)] \\
K(k) & =\bar{x}(k) \mathrm{D}^{T}\left[\mathrm{D} \bar{X}(k) \mathrm{D}^{T}+\mathrm{W}\right]^{-1} \\
\hat{x}(k) & =[I-K(k) \mathrm{D}] \bar{X}(k) \\
\bar{x}(k+1) & =\Phi \hat{x}(k)+\Delta u(k)+\bar{v}(k) \\
\bar{X}(k+1) & =\Phi \hat{X}(k) \Phi^{T}+\Omega \mathrm{N} \Omega^{T}
\end{aligned}
$$

where $\hat{x}(k)$ is the a posteriori estimate of $x(k), \bar{x}(k)$ is the a priori estimate of $x(k)$,

$$
\begin{aligned}
& \hat{X}(k)=\mathrm{E}\left\{[x(k)-\hat{x}(k)][x(k)-\hat{x}(k)]^{T}\right\} \\
& \bar{X}(k)=E\left\{[x(k)-\bar{x}(k)][x(k)-\bar{x}(k)]^{T}\right\}
\end{aligned}
$$

and $K$ is the filter gain matrix.

A very simple control law is one that gives

$$
\overline{\boldsymbol{x}}_{1}(k+1)=\left[\bar{\theta}_{\mathrm{N}}(k+1), \bar{\theta}_{\mathrm{E}}(k+1), \bar{\theta}_{\mathrm{D}}(k+1)\right]^{T}=0
$$

at every sample.

The control vector is therefore chosen as

$$
u(k)=G \hat{x}(k)-v_{0}(k)
$$

where

$$
G=-\left[\begin{array}{r:cc:c} 
& T & 0 & \\
\Phi_{11} & 0 & 0 & 0 \\
& 0 & T & \\
\hdashline 0 & 0 & 0
\end{array}\right]
$$

Now

$$
\left[\begin{array}{c}
\Delta x(k+1) \\
\tilde{x}(k+1)
\end{array}\right]=\left[\begin{array}{cc}
(I-K D) \Phi & 0 \\
\Delta \tilde{G} & \tilde{\phi}+\Delta \tilde{G}
\end{array}\right]\left[\begin{array}{c}
\Delta x(k) \\
\tilde{x}(k)
\end{array}\right]+\text { noise }
$$




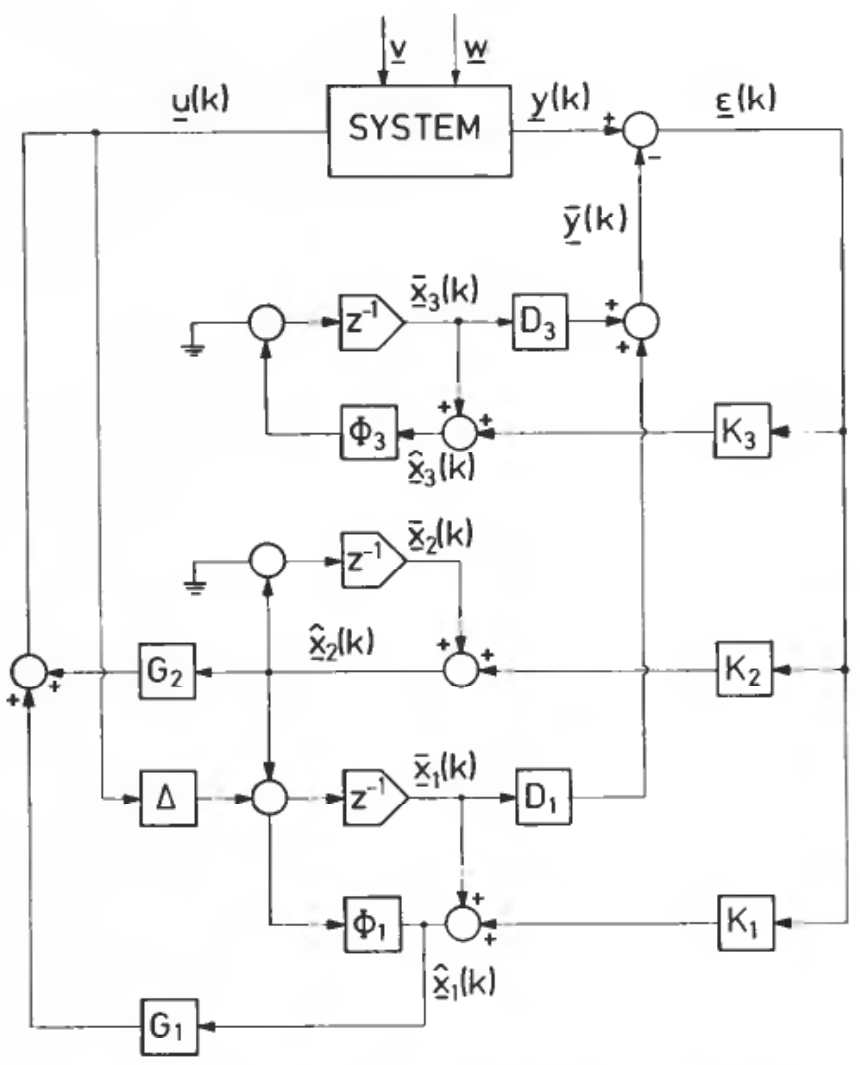

Figure 3. Block diagram of gyrocompass.

where $\Delta x=x-\hat{x}(k), \tilde{x}=\left[x_{1}^{T}, x_{4}, x_{5}\right]^{T}$ and $\tilde{\Delta} \widetilde{G}$ and $\tilde{\Phi}$ are the upper left of $\Delta G$ and $\Phi$, respectively.

We see from (22) that the eigenvalues of the system are given by the eigenvalues of $(I-K D) \Phi$ and $\tilde{\Phi}+\tilde{\Delta} \tilde{G}$.

With the control vector given by (21), $\tilde{\Phi}+\delta \tilde{G}$ will have three eigenvalues at the origin due to the reset control and two eigenvalues at $z=-1$ due to the gyro drift states.

One might expect that this reset control law would give a noisy compass error because of vessel acceleration measurement noise. However, the measurement noise is estimated and compensated for by the Kalman filter.

The compass is shown in Fig. 3.

\section{Parameter estimation}

As mentioned in $\S 3$, the model has only three unknown parameters, $L, \omega_{0}^{2}$ and $\zeta$.

The relative damping $\zeta$ in the wave model is vessel dependent, and in most cases $\zeta$ could be chosen a priori. $\omega_{0}$ could be chosen as the natural frequency of the vessel or it could be identified on-line. The latitude $L$ has to be identified on-line to detect motion in the north or south direction. 
We model the parameter vector $\theta=\left[L, \omega_{0}^{2}\right]^{T}$ as a stochastic process

$$
\theta(k+1)=\theta(k)+v_{\theta}(k)
$$

where $v_{\theta}(k)$ is zero mean white noise with covariance $V_{\theta}=E\left[v_{\theta} \boldsymbol{v}_{\theta}^{T}\right]=\operatorname{diag}\left(v_{11}, v_{22}\right)$.

A recursive least squares estimate of $\theta(k)$ is then given by (Ljung 1981, Saelid et al. 1983)

$$
\begin{aligned}
\hat{\boldsymbol{\theta}}(k+1) & =\hat{\boldsymbol{\theta}}(k)+K_{\theta}(k+1) \varepsilon(k+1) \\
K_{\theta}(k) & =P(k) \Psi^{T}(k)\left[I+\Psi(k) P(k) \Psi^{T}(k)\right]^{1} \\
P(k+1) & =\left[I-K_{\theta}(k) \Psi(k)\right] P(k)+V_{\theta}(k)
\end{aligned}
$$

Here $\hat{\theta}(k)$ is the a posteriori estimate of $\theta(k), K_{\theta}(k)$ is updating gain, $\varepsilon(k)=y(k)-\bar{y}(k)$ is the innovation, $\bar{y}(k)$ is the predicted measurement and $\Psi(k)=$ $-\partial \boldsymbol{\varepsilon}(k) / \partial \hat{\theta}$ is the sensitivity matrix. $P(k)=\mathrm{E}\left[(\boldsymbol{\theta}(k)-\overline{\boldsymbol{\theta}}(k))(\boldsymbol{\theta}(k)-\overline{\boldsymbol{\theta}}(k))^{T}\right]$ where $\overline{\boldsymbol{\theta}}(k)$ the $a$ priori estimate of $\theta(k)$.

The inclusion of the noise covariance matrix $V_{\theta}(k)$ in (25) gives the parameter estimation algorithm a tracking capability. This will generally be a better solution than a forgetting factor (Åström et al. 1977) which may result in exponential blow-up of the covariance matrix $P(k)$ in periods of little or no excitation.

A promising solution to the blow-up problem is to keep the diagonal of the covariance matrix $P(k)$ constant by an appropriate choice of $V_{0}(k)$. The algorithm will then at every sample forget as much information about each of the parameters as it receives (Saelid et al. 1983).

The sensitivity matrix $\Psi(k)=-\partial \varepsilon(k) / \partial \hat{\theta}$ can be computed from

$$
\Psi(k)=-D \frac{\partial \Delta x(k)}{\partial \hat{\theta}}
$$

where $\Delta x=\boldsymbol{x}-\overline{\boldsymbol{x}}$.

In a simulation experiment, $\omega_{0}^{2}$ was chosen a priori, and $L$ was estimated by approximating the sensitivities with

$$
D \frac{\partial \Delta x(k)}{\partial \hat{L}} \simeq D_{1} \frac{\partial \Delta x_{1}(k)}{\partial \hat{L}}
$$

where $\Delta x_{1}=x_{1}-\bar{x}_{1}=x_{1}$. From

$$
\begin{aligned}
x_{1}(k+1) & =\Phi_{11} x_{1}(k)+u(k)+v_{0}(k)+\Omega n(k) \\
u(k) & =-\hat{\Phi}_{11} \hat{x}_{1}(k)-\hat{v}_{0}(k)
\end{aligned}
$$

where $\Phi_{11}=\Phi_{11}(L), \hat{\Phi}_{11}=\Phi_{11}(\hat{L}), v_{0}=v_{0}(L)$ and $\hat{v}_{0}=v_{0}(\hat{L})$, we see that the sensitivities can be computed recursively from

$$
\frac{\partial \Delta x_{1}(k+1)}{\partial \hat{L}}=\hat{\Phi}\left[I-K D_{1}\right] \frac{\partial \Delta x_{1}(k)}{\partial \hat{L}}+\frac{\partial \hat{\Phi}}{\hat{L}}\left[I-K D_{1}\right] x(k)-\Phi \frac{\partial K}{\partial \hat{L}} D_{1} x(k)-\frac{\partial \hat{v}_{0}}{\partial \hat{L}}
$$

where $D_{1}=\left[\begin{array}{lll}g & 0 & 0 \\ 0 & g & 0\end{array}\right]$.

As $\partial K / \partial \hat{L}$ is complex to compute for time varying $K$, the term is set to zero. The results from this simulation are given in $\S 5$.

Note that the problem of parameter estimation in closed loop (Goodwin and Payne 1977) is solved by inserting (27) into (26). 


\section{Simulation}

The gyrocompass was simulated by means of the program system EXKALM in CYPROS (Tyssø 1980). The simulated system is shown in Fig. 4.

The parameters of the vessel simulator are approximated parameters for the Norwegian coastal liner 'Midnattsol' which is $104 \mathrm{~m}$ long.

Alignment from $x_{1}(0)=[-0.00005,0.0001,0.01]$ radians, that is, a compass error of $5^{\circ}$ was simulated. The vessel was stationary for $20 \mathrm{~min}(1200 \mathrm{sec}$.), and then accelerated to $10 \mathrm{~m} / \mathrm{s}$ in the east direction. Significant wave height was $1 \mathrm{~m}$. Vessel speed and course are shown in Fig. 5.

The inertial platform was simulated with white noise gyro measurement error of covariance $4 \cdot 4 \cdot 10^{-9}$, that is, no constant gyro drift. The specific force direction measurements had a covariance matrix

$$
W=\operatorname{diag}\left(1 \cdot 10^{-4}, 1 \cdot 10^{-4}\right)
$$

Latitude was $57 \cdot 3^{\circ}$ (1 radian) north.

These parameters were the simulated 'real-world' parameters. The estimator had correct values in the noise covariance matrices and correct latitude. $\omega_{0}$ in the wave estimator was chosen to $0.3 \mathrm{rad} / \mathrm{sec}$. Relative damping $\zeta$ was chosen to $0 \cdot 2$. The noise covariances in the wave estimator were

$$
\mathrm{E}\left(n_{\mathrm{N}}^{2}\right)=\mathrm{E}\left(n_{\mathrm{E}}^{2}\right)=1
$$

The initial value of $\operatorname{cov}(x)$ was assumed to be

$$
\bar{x}(0)=0.01 \operatorname{diag}(1,1,1,0,0,1,1,1,1)
$$

The compass error, $x_{3}$, is shown in Fig. 6. The error is reduced from $5^{\circ}$ to $1^{\circ}$ in $20 \mathrm{~min}$. The acceleration of the vessel after $20 \mathrm{~min}$ has little influence on the compass error.

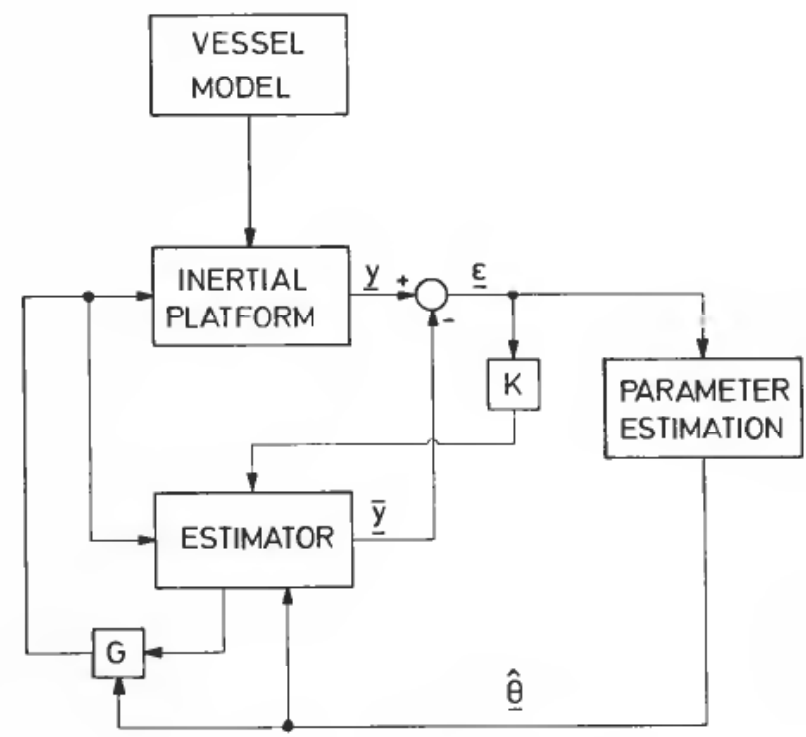

Figure 4. Simulated system. 


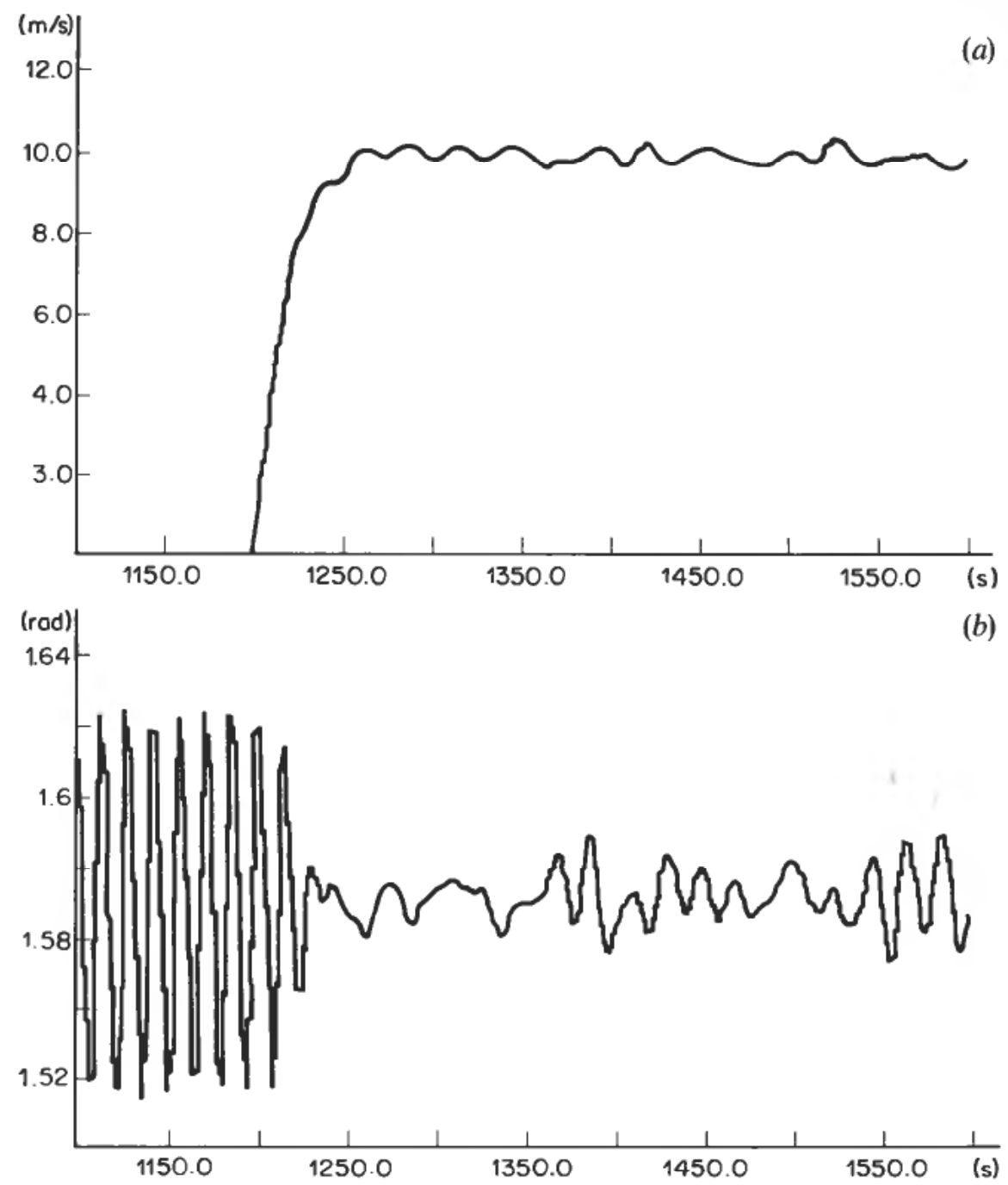

Figure 5. (a) Vessel speed first run (expanded time scale); (b) Vessel course first run (expanded time scale).

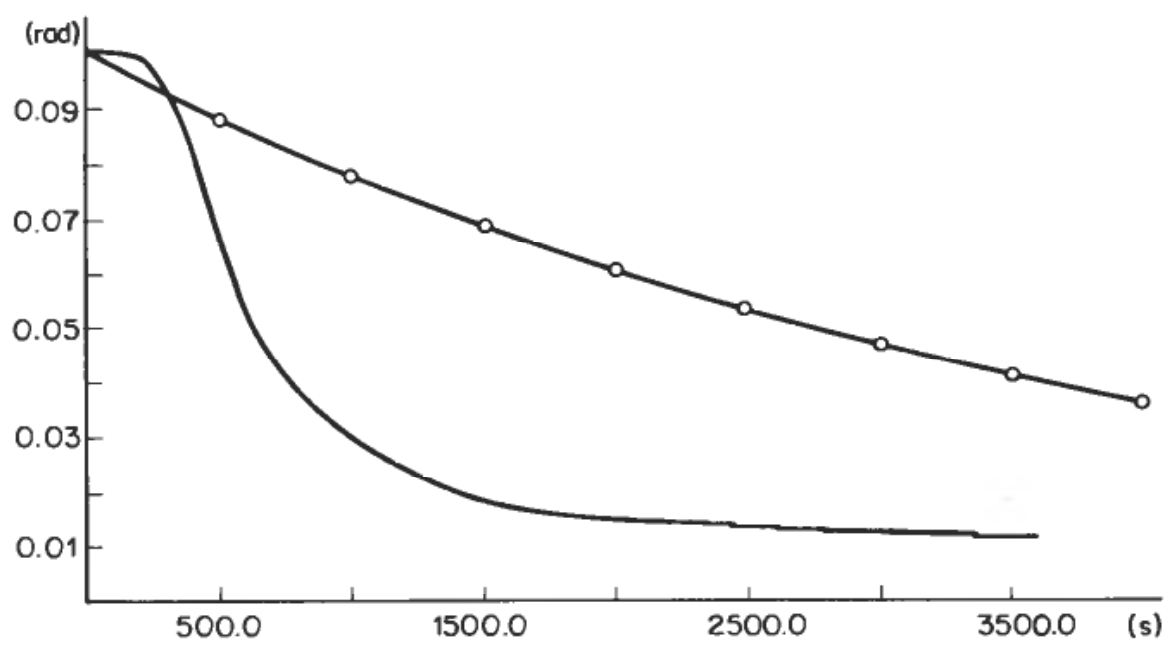

Figure 6. Compass error during alignment (solid curve) and positive convolution curve for compass error in a Sperry gyrocompass (marked with circles). 
190 O. Egeland

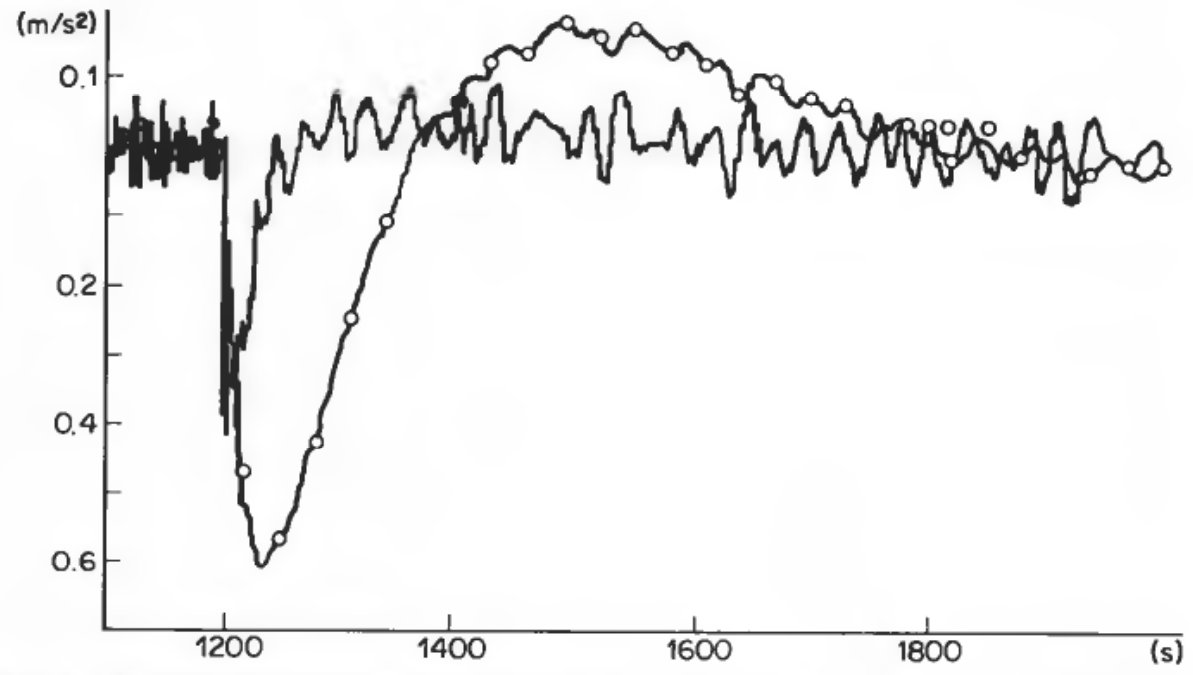

Figure 7. Estimated measurement noise (solid curve) and innovation (marked with circles) of the $y_{1}$ measurement (expanded time scale).
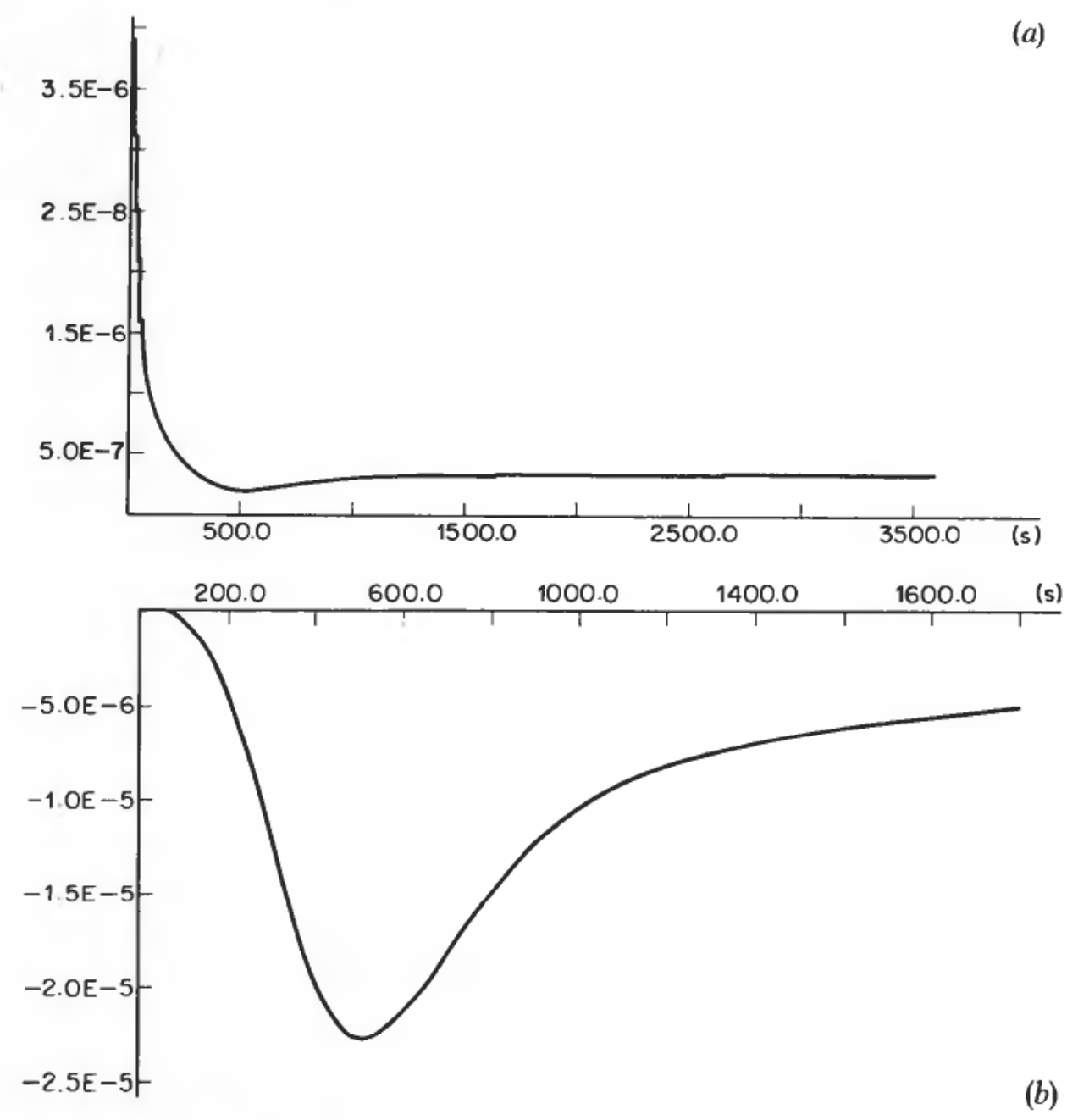

Figure 8. (a) Kalman filter gain $k_{21}$; (b) Kalman filter gain $k_{31}$. 
In a conventional Schuler tuned gyrocompass of the Sperry type, the compass error is given by

$$
\theta(s)=\frac{2 \zeta \omega_{s}}{\omega_{i e} \cos L} \frac{1}{s^{2}+2 \zeta \omega_{s} s+\omega_{s}^{2}} s^{2} L(s)
$$

where $\theta(s)$ is the Laplace transform of the compass error, $\omega_{s}$ is the Shuler frequency which corresponds to an oscillation with period $84 \mathrm{~min}$ and $\zeta$ is the relative damping of the compass (Wrigley et al. 1969).

The convolution curve for the damped oscillation of $\theta$ is shown in Fig. 6 fo $\zeta=0 \cdot 2$. We see that the conventional gyrocompass is inferior to the gyrocompass based upon multivariable control theory during alignment. Here, the effect of wave induced noise is not considered for the conventional gyrocompass. This means that the alignment of this compass will be even slower than shown in Fig. 6.

In Fig. 7, the estimated measurement noise and the innovation for the $y_{1}$ measurement is shown. We clearly see that the high frequency wave-induced noise is
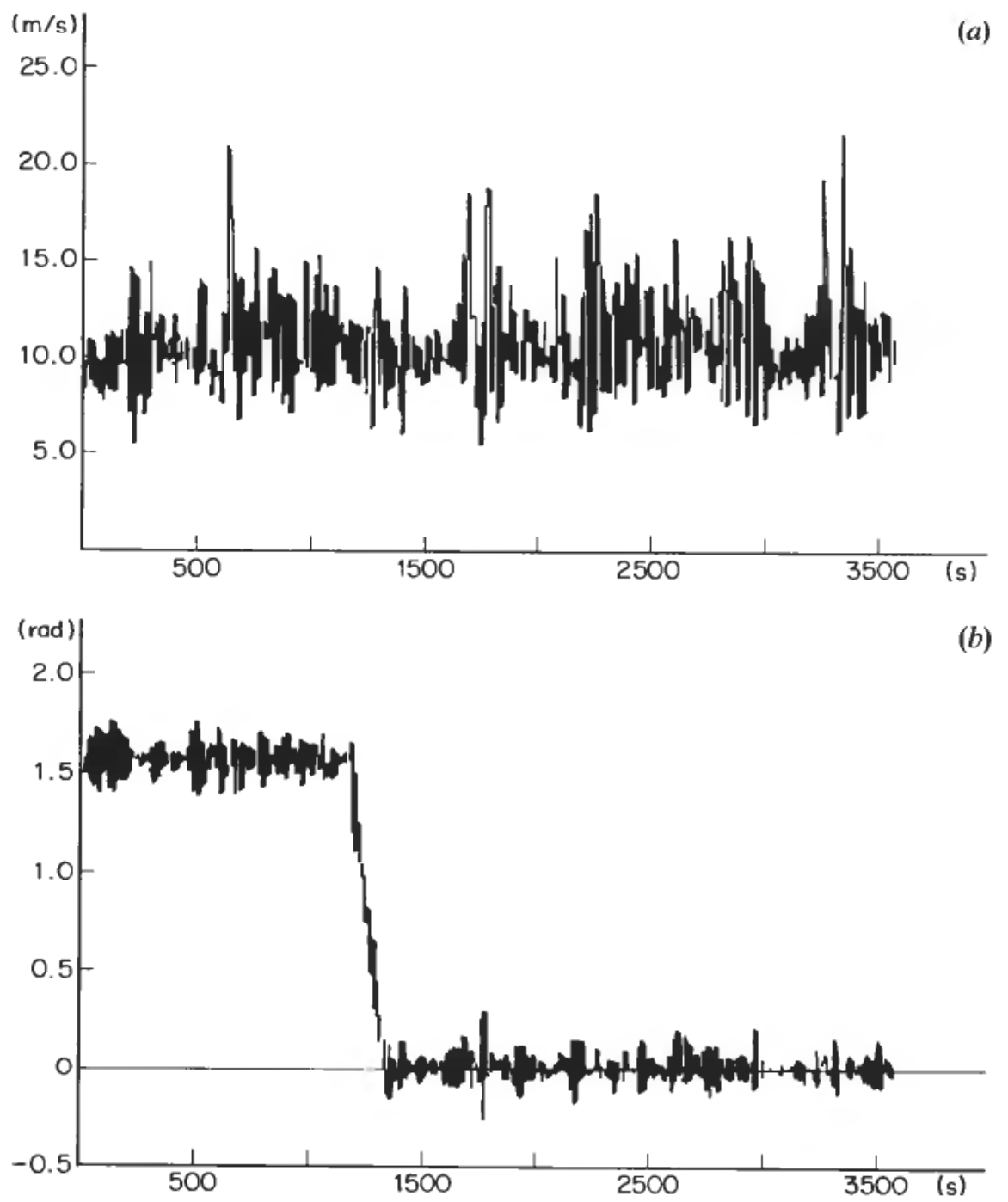

Figure 9. (a) Vessel speed second run; (b) Vessel course second run. 
estimated and compensated for, while only the high frequency part of the acceleration from 0 to $10 \mathrm{~m} / \mathrm{s}$ is compensated for.

The Kalman filter gains $k_{21}$ and $k_{31}$ are shown in Figure 8. We see that during alignment the gains are high. The gains then converge to a lower value in stationary state. As the system is linear and time-invariant, the Kalman filter gains can be pre-computed and stored, e.g. by curve-fitting.

Stationary behaviour of the compass was studied. Vessel speed was $10 \mathrm{~m} / \mathrm{s}$, the course was in the east direction with a $90^{\circ}$ change of course to the north direction after $20 \mathrm{~min}$. The compass had feedforward from vessel speed, and therefore no dynamic north phenomenon appeared (Wrigley et al. 1969). Significant wave height was $10 \mathrm{~m}$. Vessel speed and course are shown in Fig. 9.

The initial compass error was $0 \cdot 5^{\circ}$. The compass error is shown in Fig. 10. We see that the change of course after $20 \mathrm{~min}$. gives an impulse of $0.5^{\circ}$, but apart from that the compass does quite well.

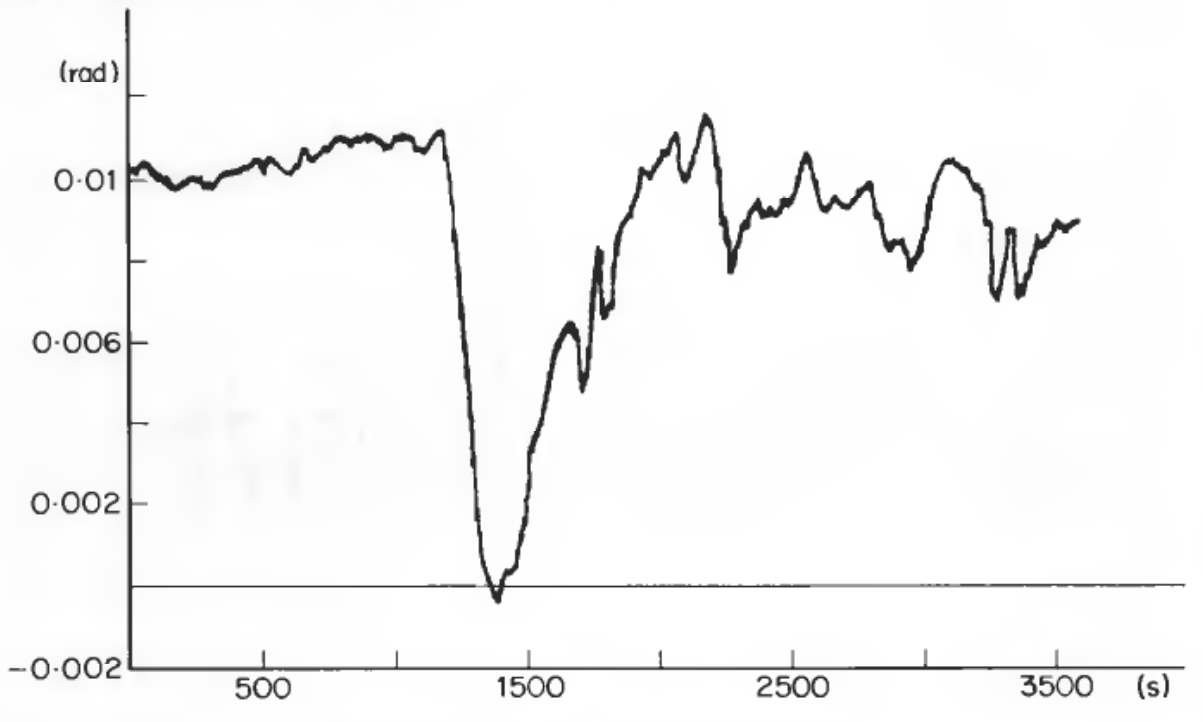

Figure 10. Compass error second run.

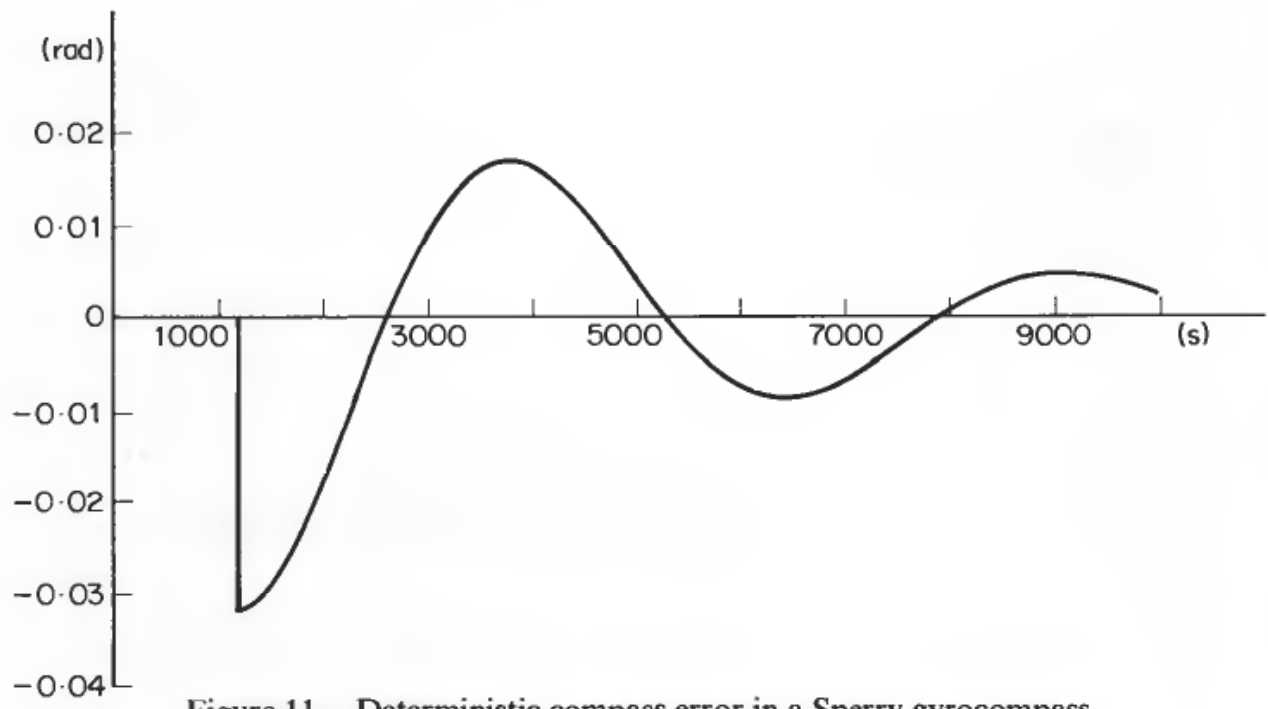

Figure 11. Deterministic compass error in a Sperry gyrocompass. 
In a conventional Sperry gyrocompass, the change of course at this speed will result in a damped oscillation as shown in Fig. 11.

Without feedforward from vessel speed, a gyrocompass will have a stationary compass error of $0.1^{\circ}$ per knot in the north direction. This means stationary error of $-2^{\circ}$ after 1200 seconds in Fig. 11. Feedforward from vessel speed can be implemented in a Sperry compass, but usually it is not.

If we add the effect of noise from waves with a significant height of $10 \mathrm{~m}$, we see that the gyrocompass based upon multivariable control theory is far better than the conventional gyrocompass.

The estimated measurement error and innovation for the $y_{2}$ measurement is shown in Fig. 12. Again we see that high frequency noise is well taken care of.

Identification of the latitude $L$ was simulated during alignment. The estimate of $L$ converged after 10 min., Fig. 13.

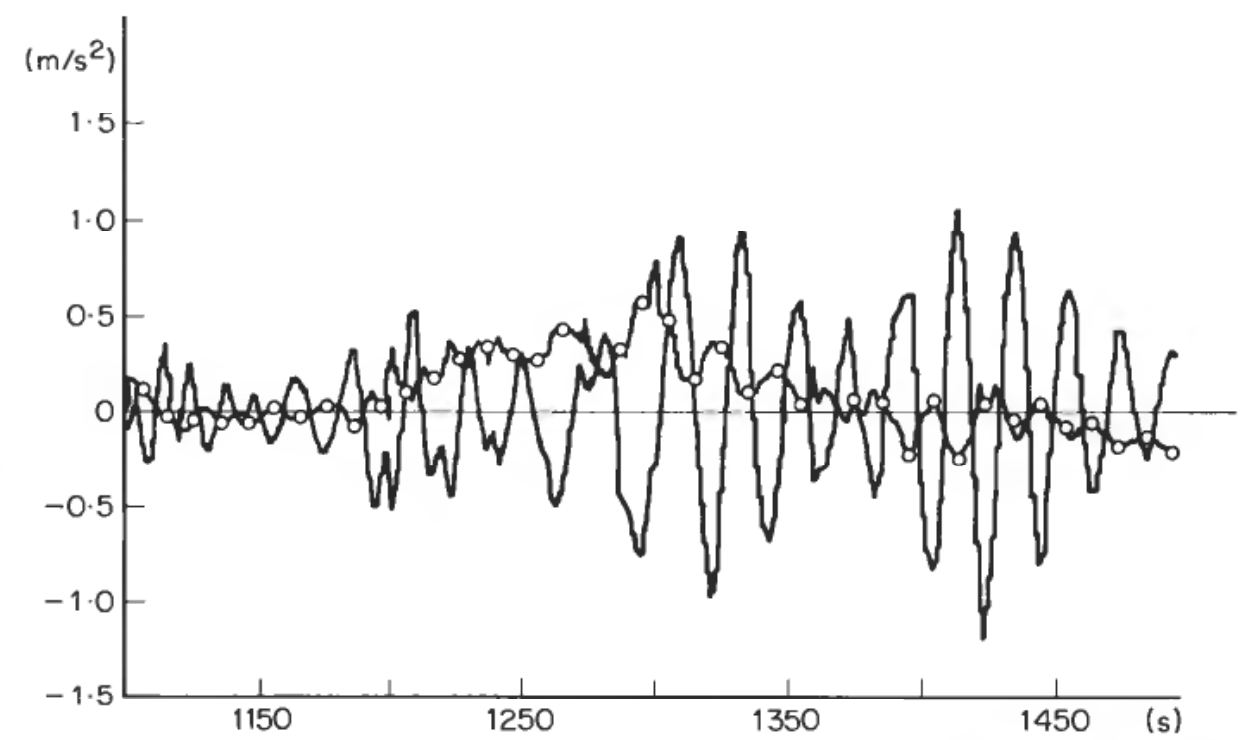

Figure 12. Estimated measurement noise (solid curve) and innovation (marked with circles) of the $y_{1}$ measurement.

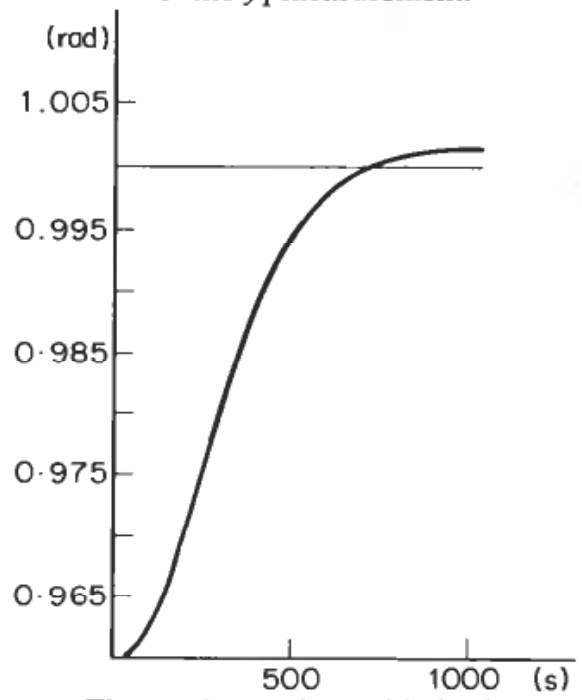

Figure 13. Estimated latitude. 


\section{Conclusions}

A gyro compass based upon multivariable control theory has been designed and simulated. Through simulation it is shown that alignment of the compass is faster than the alignment of a conventional Sperry compass. The compass also behaves well in rough sea, and the feedforward from centrifugal forces reduces the response to course changes. It is shown that the latitude can be estimated and the compass run adaptively.

\section{REFERENCES}

Åström, K. J., V. Borrisson, L. LJụng and B. WitTenMark. (1977). Theory and application of self-tuning regulators. Automatica, 13, 457.

Blanke, M., (1981). Ship Propulsion Losses Related to Automatic Steering and Prime Mover Control. Dissertation, Servolaboratory, Technical University of Denmark.

Britting, K. R., (1971). Inertial Navigation Systems Analysis. (Wiley-Interscience).

BROCK, L. D. and G. T. SCHMIDT. (1970). General questions on Kalman filtering in navigation systems. In C. T. Leondes (ed.) Theory and applications on Kalman filtering. NATO AGARD AG-139.

Ezekiel, S. and E. Knaussenberge, (1978), (eds.). Laser Inertial Rotation Sensors, Proceedings of the SPIE, 157.

Goodwin, C. G. and R. L. PAYNe. (1977). Dynamic System Identification. (Academic Press: New York).

LEONDES, C. T. (1970), (ed.). Theory and applications of Kalman filtering. NATO AGARD AG-139.

LJUNG, L. (1981). Analysis of a general recursive prediction error identification algorithm. Automatica, 71, 89-99.

MAYвeCK, P. S. (1979). Stochastic models, estimation and control, Vol. 1 (Academic Press: New York).

PrICE, W. G. and R. E. D. BISHOP (1974) Probabilistic theory of ship dynamics. (Chapman and Hall: London).

SAELID, S. and N. A. JeNSSEN. (1983). Adaptive ship autopilot with wave filter. Modeling, Identification and Control, $4,1$.

SAeLID, S., N. A. JENSSEN and J. G. BALChEN. (1983). Design and analysis of a dynamic positioning system based on Kalman filtering and optimal control. I.E.E.E. Transactions on Automatic Control, 22, 331-339.

SAELID, S. and B. Foss. (1983). Adaptive controllers with a vector variable forgetting factor. Proceedings I.E.E.E. CDC, San Antonio, 1488-1494.

SCHMIDT, G. T. (1978) (ed.). Strap-down Inertial Systems. NATO AGARD LS-95.

TYssø, A. (1980). CYPROS - cybernetic program packages. Modeling, Identification and Control, $1,1$.

WRIGLEY, W. et al. (1969). Gyroscopic Theory, Design and Instrumentation. (M.I.T. Press: Cambridge, Massachusetts). 